\title{
THE EFFECT OF DISCRETIZATION ON PARAMETER IDENTIFICATION APPLICATION TO PATIENT-SPECIFIC SIMULATIONS
}

\section{A PREPRINT}

\author{
Nava Schulmann \\ MIMESIS team \\ Inria \& Strasbourg University \\ France
}

\author{
Stephane Cotin \\ MIMESIS team \\ Inria \& Strasbourg University \\ France \\ stephane.cotin@inria.fr
}

\author{
Igor Peterlik \\ MIMESIS team \\ Inria \& Strasbourg University \\ France
}

September 2, 2019

\begin{abstract}
Identifying the elastic parameters of a finite element model from a dynamically acquired set of observations is a fundamental challenge in many data-driven medical applications going from soft surgical robotics to image-guided per-operative simulations. While various strategies exist to tackle the parameter-identification inverse problem [Aster et al., 2013], the effect of sub-optimal discretization, as often required in real-time applications, is largely overlooked. Indeed, the need to tune the parameter values in order to account for discretization-induced stiffening in specific models is reported in different works (e.g. [Chen et al., 2015, Anna et al., 2018]). However, to the best of our knowledge, no systematic study of this phenomenon exists to date, nor has any strategy to select optimal effective values been developed. Our work addresses the issue of parameter identification in coarsened meshes with special attention to the dynamical nature of the identification. We focus on the estimation of Young's modulii in simplified systems and show that the estimated stiffnesses are underestimated in a systematic manner when reducing the number of degrees of freedom. We also show that the effective stiffness of a given coarse mesh, when associated with an undersampled mesh discretization, is not constant but strongly depends on the prescribed deformations. These results show that the estimated parameters should not be considered as the true parameter value of the organ or tissue but instead are model-dependent values. We argue that Bayesian methods present a clear advantage w.r.t. classical minimization methods by their ability to efficiently adapt the local parameter values.
\end{abstract}

Keywords parameter identification · Finite element methods $\cdot$ Kalman Filters $\cdot$ Bayesian Inference $\cdot$ discretization . real-time control

\section{Introduction \& related work}

In the last decade the importance of computer medical simulation in intra-operative guidance has increased considerably. In various applications, ranging from laparoscopic liver surgery to catheter navigation or interventional radiology, physically-based numerical models play the role of complementing and regulating the information obtained by imaging. Since preoperative imaging (or three-dimensional anatomical models reconstructed from it) and intra-operative imaging often rely on different modalities, they provide different and often complementary information on tissue structures as well as deformation changes. In that case, the fusion of intra-operative and pre-operative images into a unique coordinate frame adds significant value. This can provide an enhanced visualization, for instance of the subsurface anatomy during laparoscopy, to help determine resection planes and facilitate navigation [Heiselman et al., 2017]. Generally speaking, intra-operative image augmented with pre-operative data results in easier and better guidance of an intervention, leading to reduced invasiveness and/or increased accuracy during surgical procedures [Peters and Cleary, 2008]. In 
percutaneous ablations and tumor resections, augmented intra-operative images can facilitate the localization of small tumors. In this case, the augmented image helps follow the safety margins near the tumor while preserving a sufficient amount of healthy tissue.

However, one challenge that often hinders the ability to fuse pre- and intra-operative images comes from the tissue deformations that often take place between the two image acquisitions. Handling these deformations requires nonrigid registration methods to align the pre-operative models or images onto the intra-operative images. A very large body of work has addressed this problem, and it has been shown that biomechanical models can achieve excellent results even in the presence of large deformations ([Haouchine et al., 2015] [Collins et al., 2017]).

If a real-time augmentation of the data is sought, to provide augmented reality as in ([Haouchine et al., 2015] or [Suwelack et al., 2014]), this brings two strong constraints onto the soft tissue model. The predictive accuracy of the model should be higher than what is generally used in the context of computer-based training systems, while still being under the constraint of real-time computation. Achieving these objectives jointly is a challenge, and generally requires some trade-off. In addition, intra-operative guidance requires specific models, tailored to the patient. This is necessary to ensure simulations that are as accurate as possible. While the general constitutive law for a given organ is known, its parameters are often patient-specific. In this paper we address an important issue that stems from the association of these constraints: real-time computation, patient-specific modeling, and biomechanical modeling.

Patient-specific models can be obtained, at least partially, through pre- or intra-operative imaging. While the geometry of the organ can be extracted quite easily, and almost automatically, through pre-operative images such as computed tomography (CT) or magnetic resonance imaging (MRI), material properties are a lot more difficult to determine in a patient-specific way. In some cases, these parameters may be obtained by elastography [Ophir et al., 1991], a technique that relies on MRI or ultrasound imaging modalities. However, this solution remains limited today in a clinical context due its technical requirements. For this reason, the development of alternative identification methods, that allow for real-time parameter estimation from per-operative images, has significantly increased in the recent years (see for example [Avril and Evans, 2017]).

Though different numerical approaches for resolving the mechanical behaviors of soft tissues exist, finite element (FE) methods are by far the most commonly used in the medical simulation and biomechanics communities (see [Han et al., 2011] and [Zhang et al., 2018] for instance). Different approaches have been proposed to reduce the high computational cost of FE methods in order achieve real-time performances. A first strategy consists of replacing the complex nonlinear physical laws by linear descriptions. this solution, however, is rarely appropriate for anatomical structures. Another direction is to perform model order reduction such as Proper Orthogonal Decomposition (POD) [Bialecki et al., 2005] or Proper Generalized Decomposition (PGD) [Niroomandi et al., 2013]. These techniques allow for a significant computational gain, but at the expense of a pre-computation step which can be very costly. When this is not possible due to time constraints, as it can be the case for patient-specific simulations in a clinical context, alternative strategies are used. A common approach consists of maintaining the complexity of the physical model while reducing the number of degrees of freedom in the mesh. The solution obtained in this case is therefore only an approximation of the solution at convergence.

Reducing the FE mesh resolution can also have a substantial impact on the results of parameter identification. Parameter values may not converge to ground-truth values, but strongly depend on the level of discretization [Aster et al., 2013]. For example, coarse meshes tend to be stiffer than fine meshes (see 1). Different authors have suggested to resolve this discretization-induced parameter dependency by tuning the parameter values in order to fit the observation (e.g. [Chen et al., 2015, Anna et al., 2018]). However, neither a systematic study of this phenomenon exists, nor a strategy to select optimal effective values has been developed. More importantly, simple tests show that stiffening depends not only on the coarsening level, but also on the type of deformation. In other words, for a given model, estimated parameters that fit the observations within a certain range of deformation, may give rise to important errors when used to simulate different manipulations, as illustrated in Figure 1.

The goal of this work is to study this effect systematically and suggest a general strategy for an efficient dynamical parameter identification in coarse meshes. We will discuss the simulation framework and the optimization scheme in Section 2, experimental in-silico results are reported in Section 3 We conclude with a summary and discussion of future work.

\section{Methodology}

As stated in the introduction, in this work, we are interested in simulations of large deformations occurring during manipulations with soft tissues, e.g. during a laparoscopic surgery. Models for this type of simulations have been developed in the theory of elasticity and are parametrized by material properties. 

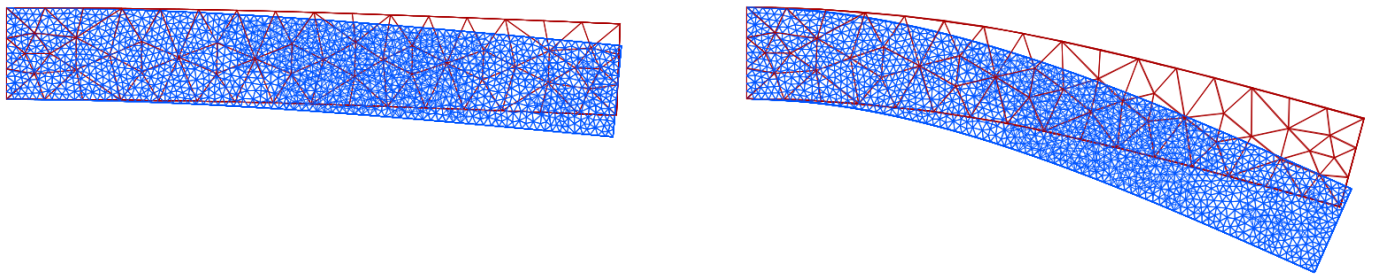

Figure 1: Comparison between the deformations of fine and coarse meshes under small and big mechanical stresses. The fine mesh (blue) consists of 11355 nodes and the coarse mesh (red) of 239 nodes. Each sub-figure shows the deformations of the two meshes under the same stress constraints. In both cases the coarse mesh presents a stiffer behavior than the fine mesh, however, the stiffening is clearly higher when the stress is more important.

The estimation of material properties is typically composed of three phases: (i) selection of appropriate parametric model which captures the behaviour of interest; (ii) experimental measurements typically performed on representative samples; (iii) parameter fitting: an optimization procedure which yields values of the selected model so that the outputs of the model correspond to the measurements.

In the context of tissue modeling for computer-aided interventions, each of the three phases listed above gives rise to specific challenges: models capturing the behaviour of tissues are highly non-linear, the accuracy of measurements is quite limited since they must be performed either on cadavers or non-invasively, parameters obtained by fitting are often difficult to generalize since biomechanical coefficients of tissues show high inter-patient variability.

Being aware of these challenges, many of which have been studied in the literature [Marchesseau et al., 2010, Rifai et al., 2011, Wittek et al., 2009], we focus solely on the problematic of parameter identification due to coarse discretization which is necessary in real-time context. To this end, we propose a methodology based on numerical experiments which were designed to be generic while maintaining the essential features related to real-time parameter identification in coarse meshes, as explained below.

\subsection{Finite-element Modeling}

In order to investigate the solution of the problem introduced by the dependency of parameters on FE mesh density, we employ a model of highly-deformable homogeneous object having a simple regular geometry. The behaviour of the cantilever beam, fixed on one side and subjected to uniformly-distributed pressure, is modelled with StVenant-Kirchhoff hyperelastic constitutive law. The resulting boundary-value problem solved by the finite element method where linear tetrahedral elements (usually denoted as P1) are employed to discretize the domain of the object.

This type of elements is usually not recommended because of its high discretization error (as stress and strain are constant over the volume of an element) and locking phenomenon. However, here we must recall the the real-time context of this work which puts a hard constraint on the computationalal time. From this point of view, P1 elements are optimal since they require significantly lower computational time needed to assemble the element equations when compared to higher-order and/or non-simplex elements which require advanced quadratures to perform the integration. While also in the case of P1 elements, their number used for the discretization is already limited by the real-time requirement, in the case of more complex elements, the resolution of the mesh would not allow for a reasonable approximation of the modelled object.

\subsection{Identification method}

In order to account for the dynamic nature of data acquisition and for the parameter dependencies on the prescribed deformation, a certain form of adaptive filtering is needed. Classical optimization methods minimize some metrics between the model predictions and the data. The method of least squares (LS) is a standard approach in which the overall solution minimizes the sum of the error squares. Recursive methods also known as adaptive filters allow for a dynamical update of the parameter estimation. They split into Stochastic gradient filters (different variants of LS) and least squares recursive filter (RLS). In the case of time independent parameters and in the presence of large amount of data, the different identification methods converge to the same values, thus the choice between the filters is a tradeoff 
between efficiency and computational costs. However, when data is sparse, the convergence rates may highly affect the estimations. In order to tackle the data sparsity in the context of computer-aided interventions, we employ Bayesian methods, namely Kalman filters which present particularly good convergence rates and have proven their worth in numerous real-time applications. Bayesian methods take into account some a priori knowledge about the state of the system and combine it with measured data to infer an estimated state distribution. In this framework, the parameter identification is performed through a joint estimation: the system state is augmented by the parameters of the model, and the filter simultaneously updates the system state together with the parameters. This method may be interpreted as a generalized LMS where, instead of following a local gradient, a path which optimizes the global convergence is chosen.

The original Kalman filters, which were designed for linear systems, have been adapted to non-linear estimation. While Extended Kalman filter requires explicit derivative of the model w.r.t. the parameter which cannot be obtained easily in our case, we opt for Unscented Kalman filter (UKF). In a nutshell, UKF samples the probability space in each step of the estimation and thus requires forward execution of the model (i.e., FE simulation) for each sample. This approach would become computationally infeasible in the case of joint state-parameter estimation due to a very high number of samples (thus FE simulations), we rely on reduced-order version of this filter (denoted as RO-UKF) proposed by [Moireau and Chapelle, 2011]: in our case, only the samples related to the model parameters are propagated through the non-linear FEM.

\section{Results}

In this section, we report the results of our in-silico deformation experiments. We first explore the dependency between the resolution level and the estimated stiffness for one given deformation. Then, for an intermediate coarsening level, we compute the apparent stiffnesses for increasing pressures in various directions. We finally compare the shape errors between simulated objects, with and without data assimilation.

\subsection{Estimated Stiffness}

\subsubsection{Element size dependence}

To assess the effect of coarse meshing on parameter identification, we impose identical bending stresses to geometrically identical beams, which are modeled with different levels of discretization. The Young's modulus of each beam is estimated using a Kalman Filter. As can be seen in Figure 2, the estimated values asymptotically approach the ground truth value of $5000 \mathrm{kPa}$ when the resolution is high; however, under-sampling the mesh leads to a systematic underestimation of the stiffness. These results align well with our expectations: coarse meshes tend to present a stiffer elastic behavior than fine meshes. Accordingly, the filter 'softens' the Young's modulus of coarse meshes, in order to fit the observations.

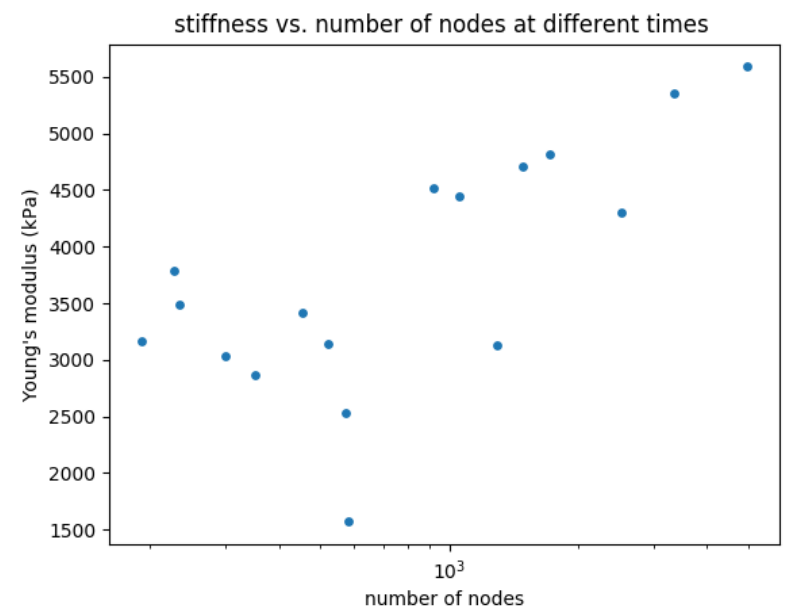

Figure 2: Young's modulus of an homogeneous beam as a function of mesh resolution, as estimated by data assimilation. The values are underestimated for coarse meshes and approach the ground-truth value at high resolutions 
Two important facts are highlighted by this simple experiment: first, in order to obtain realistic deformations with undersampled meshes, it is necessary to tune the Young's modulus, to compensate the discretization-induced stiffening. More generally, these results indicate that in the case of under-sampled meshes, the process of parameter identification does not give access to true parameter values of the elastic object. Rather, the estimated parameters are effective values 'chosen' by the filter to optimally match between the given, inaccurate model, and the observations.

\subsubsection{Deformation strength and direction dependence}

The results reported in the last paragraph suggest that it is possible to offset the discretization effects by carefully selecting the model parameters. However, another series of experiments reported in Figure 4 shows that things are not so simple. Here again we estimate the Young's modulus using a Kalman filter, but this time the applied pressure is varied for a fixed discretization. Figure 4 a. shows the estimated stiffness as a function of time, in a quasi-static process, for stretching and/or bending deformations in different directions. Comparing to the ground-truth value of $5000 \mathrm{kPa}$, we see that the value of Young's modulus is not constant. The 'local' stiffness strongly depends on the direction and strength of the deformation. To highlight the spatial dependencies, the same data is presented as a heat map in Figure 4 4 . The positions of the data points correspond to the positions of one node of the beam, at different stages of the deformation. The color of each point represents the local value of the estimated stiffness. These results have an important implication in our application context: when using a coarse mesh in a real-time medical simulation, estimating the patient-specific parameters at the beginning of the intervention may not be sufficient. Parameters that fit the observations within a certain range of deformations may cause important errors when used to simulate different manipulations.

\subsection{Shape Error}

We have shown that a parameter identification procedure in coarse meshes gives rise to values that significantly differ from ground-truth values. Moreover, the estimated parameters not only depend on the mesh resolution but also on the nature of the deformation.

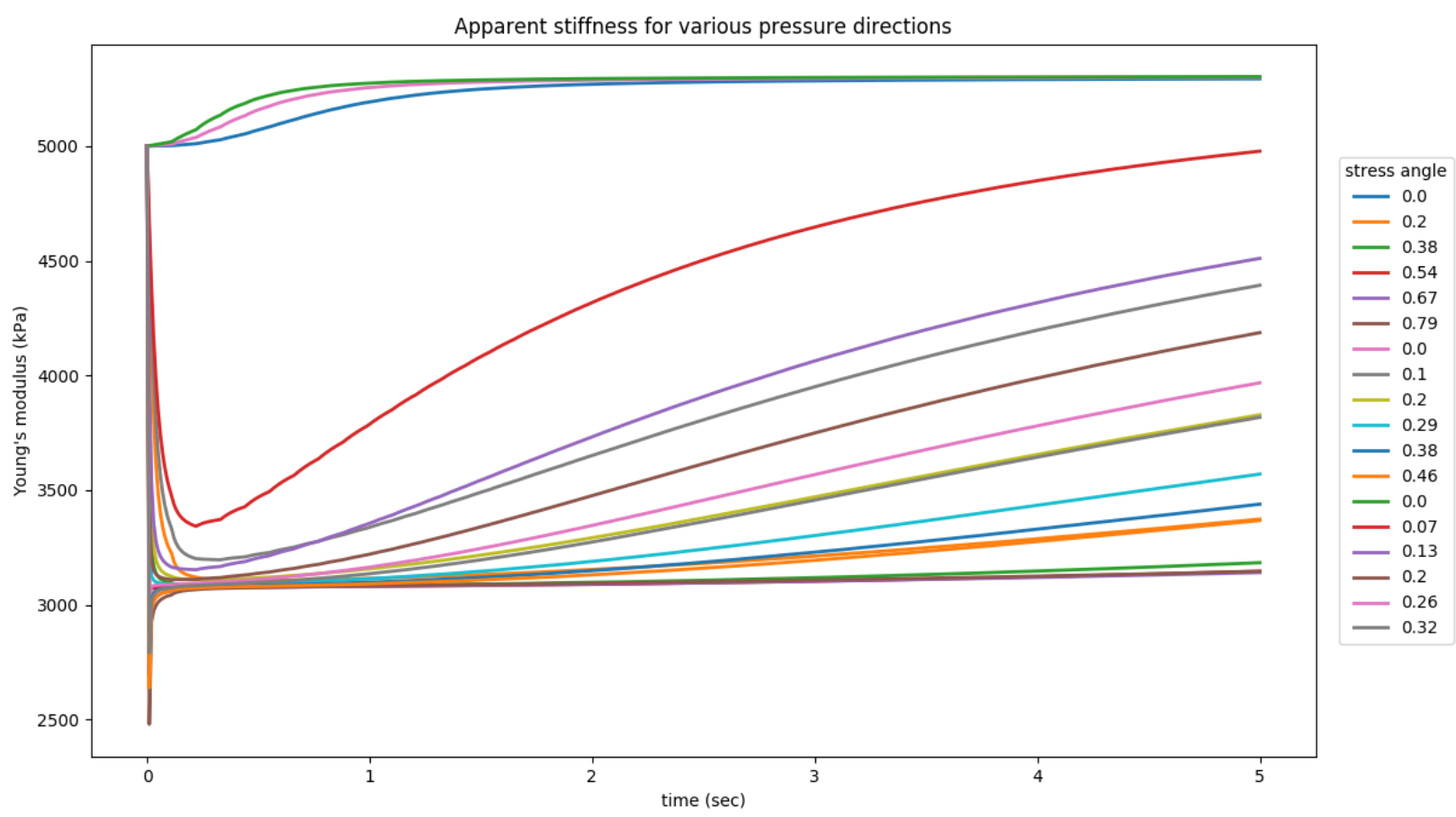

Figure 3: Effective stiffness dependence on deformation direction and strength. Effective stiffness as a function of time in a quasi-static process where pressure strength is regularly increased. Different colors correspond to different stress directions.

This results raise a fundamental question: is there a sense in estimating parameters that are not constant but vary as a function of the system state? Does this heavy procedure contribute to the quality of the simulations? In order to answer 
this question, we compare in this section the simulation predictions with and without data assimilation. Using a regular grid we measure the shape error of each deformed system, the shape error being defined as the sum of the euclidean distances of the grid's nodes from the nodes of the reference system.

Figure 4 sketches the shape error for different levels of discretization. Red points represent the shape errors for the deformations obtained by direct simulations, using the ground-truth value of the Young's modulus. Green points correspond to the shapes attained by data assimilation. Apart from two system where the filtering process did not converge, these results clearly demonstrate the efficiency of the data assimilation process in reducing the shape errors.

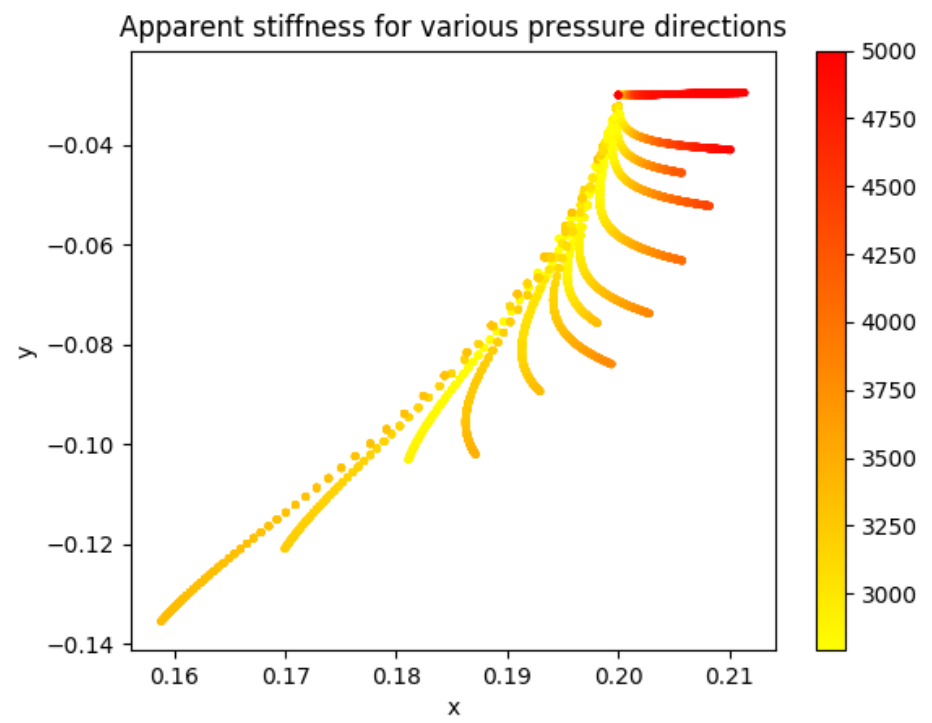

Figure 4: Effective stiffness dependence on deformation direction and strength represented as a heat map in the deformation space. All data points correspond to data assimilation estimations obtained for a coarse mesh of 510 nodes. Ground-truth is simulated with a fine mesh of 11394 nodes.

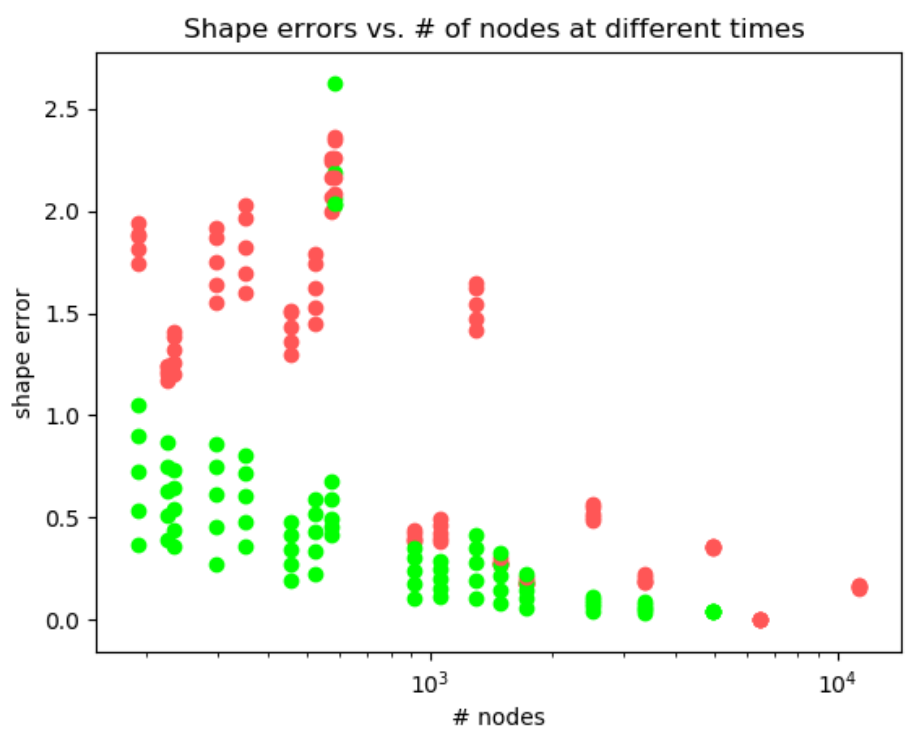

Figure 5: Shape error as a function of mesh resolution (number of nodes) for direct (red) and data assimilation (green) simulations. The error decreases with resolution level. In coarse meshes errors are significantly reduced by data assimilation. 


\section{Discussion}

In this paper, we studied the effect of mesh resolution on the effective stiffness of an elastic solid, in the context of real-time simulation and parameter estimation. We have shown that the estimated stiffness depends on the discretization level but also on the deformation. We also demonstrated that Kalman filters yield smaller shape errors than direct simulations parameterized with ground-truth values.

This means, among other things, that when insufficiently fine meshes are used, the estimated parameters should not be considered as the true parameter values of the organ or tissue, but instead as effective parameters that allow retrieving a shape that fits the observations. The values which are estimated can be seen as 'local', in the sense that they depend on the prescribed deformation. If the simulation is performed in real-time, and an efficient Kalman filter is used (such as a Reduced Order Unscented Kalman Filter), the filter might be able to continuously estimate a new parameter set that will allow the model to fit the data. However, if observations were to disappear then the model will start drifting from the real shape.

A clear strategy regarding parameter estimation in the interventional context arises from these findings. First, parameters should not be determined once and then used by the simulation (as it would be the case with an optimization method) but rather estimated constantly along the intervention. Secondly, a special attention should be given to the convergence rate of the identification. If in principle, any type of adaptive filter may allow for local parameter identification, in the case of interventional therapy, where little data is available at each step of the deformation, slow convergence rates may highly deteriorate the simulated prediction. Accordingly, despite their computational cost, Bayesian filters present a clear advantage w.r.t classical ones due to their highly efficient convergence.

\section{References}

[Anna et al., 2018] Anna, M., Carton, A.-K., Muller, S., and Payan, Y. (2018). Breast biomechanical modeling for compression optimization in digital breast tomosynthesis. In Gefen, A. and Weihs, D., editors, Computer Methods in Biomechanics and Biomedical Engineering, Lecture Notes in Bioengineering, pages 29-35. Springer International Publishing.

[Aster et al., 2013] Aster, R. C., Borchers, B., and Thurber, C. H. (2013). Parameter estimation and inverse problems (second edition. Academic Press, second edition.

[Avril and Evans, 2017] Avril, S. and Evans, S. (2017). Material Parameter Identification and Inverse Problems in Soft Tissue Biomechanics, volume 573.

[Bialecki et al., 2005] Bialecki, R. A., Kassab, A. J., and Fic, A. (2005). Proper orthogonal decomposition and modal analysis for acceleration of transient fem thermal analysis. International Journal for Numerical Methods in Engineering, 62:774-797.

[Chen et al., 2015] Chen, D., Levin, D. I. W., Sueda, S., and Matusik, W. (2015). Data-driven finite elements for geometry and material design. ACM Trans. Graph., 34(4):74:1-74:10.

[Collins et al., 2017] Collins, J. A., Weis, J. A., Heiselman, J. S., Clements, L. W., Simpson, A. L., Jarnagin, W. R., and Miga, M. I. (2017). Improving registration robustness for image-guided liver surgery in a novel human-to-phantom data framework. IEEE transactions on medical imaging, 36(7):1502-1510.

[Han et al., 2011] Han, L., Hipwell, J. H., Tanner, C., Taylor, Z., Mertzanidou, T., Cardoso, J., Ourselin, S., and Hawkes, D. J. (2011). Development of patient-specific biomechanical models for predicting large breast deformation. Physics in Medicine and Biology, 57(2):455-472.

[Haouchine et al., 2015] Haouchine, N., Cotin, S., Peterlik, I., Dequidt, J., Lopez, M. S., Kerrien, E., and Berger, M.-O. (2015). Impact of soft tissue heterogeneity on augmented reality for liver surgery. IEEE transactions on visualization and computer graphics, 21(5):584-597.

[Heiselman et al., 2017] Heiselman, J. S., Clements, L. W., Collins, J. A., Weis, J. A., Simpson, A. L., Geevarghese, S. K., Kingham, T. P., Jarnagin, W. R., and Miga, M. I. (2017). Characterization and correction of intraoperative soft tissue deformation in image-guided laparoscopic liver surgery. Journal of Medical Imaging, 5(2):021203.

[Marchesseau et al., 2010] Marchesseau, S., Heimann, T., Chatelin, S., Willinger, R., and Delingette, H. (2010). Multiplicative jacobian energy decomposition method for fast porous visco-hyperelastic soft tissue model. In International Conference on Medical Image Computing and Computer-Assisted Intervention, pages 235-242. Springer.

[Moireau and Chapelle, 2011] Moireau, P. and Chapelle, D. (2011). Reduced-order unscented kalman filtering with application to parameter identification in large-dimensional systems. ESAIM: Control, Optimisation and Calculus of Variations, 17(2):380-405. 
[Niroomandi et al., 2013] Niroomandi, S., González, D., Alfaro, I., Bordeu, F., Leygue, A., Cueto, E., and Chinesta, F. (2013). Real-time simulation of biological soft tissues: a pgd approach. International Journal for Numerical Methods in Biomedical Engineering, 29(5):586-600.

[Ophir et al., 1991] Ophir, J., Céspedes, I., Ponnekanti, H., Yazdi, Y., and Li, X. (1991). Elastography: A quantitative method for imaging the elasticity of biological tissues. Ultrasonic Imaging, 13(2):111 - 134.

[Peters and Cleary, 2008] Peters, T. and Cleary, K. (2008). Image-guided interventions: technology and applications. Springer Science \& Business Media.

[Rifai et al., 2011] Rifai, K., Cornberg, J., Mederacke, I., Bahr, M. J., Wedemeyer, H., Malinski, P., Bantel, H., Boozari, B., Potthoff, A., Manns, M. P., et al. (2011). Clinical feasibility of liver elastography by acoustic radiation force impulse imaging (arfi). Digestive and Liver Disease, 43(6):491-497.

[Suwelack et al., 2014] Suwelack, S., Röhl, S., Bodenstedt, S., Reichard, D., Dillmann, R., dos Santos, T., Maier-Hein, L., Wagner, M., Wünscher, J., Kenngott, H., et al. (2014). Physics-based shape matching for intraoperative image guidance. Medical physics, 41(11):111901.

[Wittek et al., 2009] Wittek, A., Hawkins, T., and Miller, K. (2009). On the unimportance of constitutive models in computing brain deformation for image-guided surgery. Biomechanics and modeling in mechanobiology, 8(1):77-84.

[Zhang et al., 2018] Zhang, J., Zhong, Y., and Gu, C. (2018). Deformable models for surgical simulation: A survey. IEEE Reviews in Biomedical Engineering, 11:143-164. 